\title{
Crossmodal interaction between the mental number line and peripersonal haptic space representation in sighted and blind individuals
}

\author{
Zaira Cattaneo \\ University of Milano-Bicocca, Milan, Italy \\ Micaela Fantino \\ University of Pavia, Pavia, Italy \\ Carla Tinti \\ University of Turin, Turin, Italy \\ Juha SiLvanto \\ University College London, London, England \\ and Helsinki University of Technology, Espoo, Finland \\ AND \\ Tomaso VecchI \\ University of Pavia, Pavia, Italy
}

\begin{abstract}
Humans tend to represent numbers in the form of a mental number line. Here we show that the mental number line can modulate the representation of peripersonal haptic space in a crossmodal fashion and that this interaction is not visually mediated. Sighted and early-blind participants were asked to haptically explore rods of different lengths and to indicate midpoints of those rods. During each trial, either a small (2) or a large (8) number was presented in the auditory modality. When no numbers were presented, participants tended to bisect the rods to the left of the actual midpoint, consistent with the notion of pseudoneglect. In both groups, this bias was significantly increased by the presentation of a small number and was significantly reduced by the presentation of a large number. Hence, spatial shifts of attention induced by number processing are not limited to visual space or embodied responses but extend to haptic peripersonal space and occur crossmodally without requiring the activation of a visuospatial representation.
\end{abstract}

Consistent research has shown that the spatial representation of numbers interacts with the perception of visual space (for a review, see Umiltà, Priftis, \& Zorzi, 2009; Wood, Willmes, Nuerk, \& Fischer, 2008). Much of the evidence has come from studies using the line bisection task, a task that is widely used for investigating the allocation of spatial attention (Jewell \& McCourt, 2000). In this task, a leftward spatial bias has been observed when the bisected line consisted of series of small digits, and a rightward bias has been observed for lines consisting of series of large digits (Fischer, 2001; see also de Hevia, Girelli, \& Vallar, 2006). Similarly, in a study of left-neglect patients (Bonato, Priftis, Marenzi, \& Zorzi, 2008), the bisection error was shifted contralesionally when the digit 1 was presented at each end of the to-be-bisected visual line, whereas it was shifted ipsilesionally when the digit 9 was presented. It has also been reported that priming by large numbers eliminates pseudoneglect (i.e., the tendency to perceive a stimulus midpoint to the left of its actual physical midpoint; cf. Bowers \& Heilman, 1980) in visual line bisection (Cattaneo, Silvanto, Pascual-Leone, \& Battelli, 2009). Finally, numerical magnitude has been found to affect responses in a task requiring grayscale judgments that did not require any overt or covert left/right responses (Nicholls, Loftus, \& Gevers, 2008), ruling out explanations that are based solely on aspects of response selection.

The studies reviewed above all involved the visual modality only (i.e., visual judgments of spatial features).

Z.Cattaneo, zaira.cattaneo@unimib.it 
Nonetheless, spatial-numerical association of response codes (SNARC)-type effects (i.e., faster right-hand responses for large numbers and faster left-hand responses for small numbers; for a review, cf. Dehaene, Bossini, \& Giraux, 1993), which are usually observed with visually presented numbers, have also been reported with numbers presented in the auditory modality (e.g., Castronovo \& Seron, 2007; Nuerk, Wood, \& Willmes, 2005; Wood, Nuerk, \& Willmes, 2006). This suggests that the association between numerical magnitude and hand-based space is modality independent. In fact, attentional shifts due to number magnitude can also occur crossmodally: In particular, Brozzoli et al. (2008) showed that visual presentation of a number $(1,5)$ modulated the detection threshold of vibratory tactile stimuli delivered on the little finger or the thumb and that the direction of this effect depended on whether the hand was in a palm-up or palm-down posture. Their results suggest that, although individuals can use both space- and body-based representations of numbers, the former prevails when the two are in conflict. Notably, in Brozzoli et al.'s experiment, the connection between sensory processing and the mental number line was investigated by manipulating which fingers participants used in counting without requiring any explicit judgment on peripersonal space. From this study, it is therefore not clear whether the mental number line can modulate the perception of peripersonal space in a crossmodal fashion.

The aim of the present research was to investigate whether the mental number line can modulate the perception of peripersonal space in a crossmodal fashion by testing whether auditorily presented numbers affect performance in a haptic rod-bisection task. It is important to note that we tested both sighted (blindfolded) individuals and early-blind individuals. The latter were included in order to investigate whether the possible crossmodal influence of auditorily presented numbers on haptic spatial judgments requires the mediation of a visuospatial mental representation. In fact, there is evidence that crossmodal interactions occur only when sensory information from different modalities is remapped onto an external spatial reference frame, and this may need vision in order to develop (see Collignon, Voss, Lassonde, \& Lepore, 2009; Putzar, Goerendt, Lange, Rösler, \& Röder, 2007; Röder, Föcker, Hötting, \& Spence, 2008; Röder, Kusmierek, Spence, \& Schicke, 2007; Röder, Rösler, \& Spence, 2004). Previous evidence suggests that blind individuals represent numerical magnitude along the mental number line: They display the SNARC effect when numbers are presented in the auditory modality and also show other classic properties of normal number representation, such as the distance effect (i.e., longer reaction times when discriminating numerically close quantities, as compared with more distant quantities) (Castronovo \& Seron, 2007; Szücs \& Csépe, 2005). However, it is not known whether the mental number line, when activated, can influence haptic estimation of external space in blind individuals who usually encode space in body-reference coordinates without generating a visuospatial mental representation (Röder et al., 2008; Röder et al., 2007; Röder et al., 2004).
Table 1

Details of the Blind Participants

\begin{tabular}{cccll}
\hline Participant & Sex & Age & \multicolumn{1}{c}{ Etiology } & $\begin{array}{c}\text { Blindness } \\
\text { Onset }\end{array}$ \\
\hline 1 & M & 39 & Optic nerve damage & Birth \\
2 & F & 39 & Optic nerve damage & Birth \\
3 & F & 35 & Optic nerve damage & Birth \\
4 & F & 43 & Oxygen therapy & Birth \\
5 & M & 22 & Bilateral retinoblastoma & Birth \\
6 & M & 37 & Optic nerve damage & Birth \\
7 & M & 33 & Trauma & 4 months \\
8 & M & 40 & Oxygen therapy & Birth \\
9 & M & 46 & Optic nerve damage & Birth \\
10 & F & 29 & Retrolental fibroplasias & Birth \\
11 & M & 48 & Optic nerve damage & Birth \\
12 & M & 46 & Optic nerve damage & Birth \\
13 & M & 32 & Trauma & Birth \\
14 & M & 23 & Optic nerve damage & Birth \\
15 & F & 40 & Trauma & 6 months \\
16 & F & 28 & Trauma & 8 months \\
17 & F & 38 & Optic nerve damage & Birth \\
\hline
\end{tabular}

Note-All of the blind participants were proficient in Braille.

\section{METHOD}

\section{Participants}

Seventeen congenitally or early-blind participants (10 males; mean age $=36)($ see Table 1 for details $)$ and 23 sighted participants ( 8 males; mean age $=27$ years) took part in the experiment. All of the participants were right-handed, as assessed by the Edinburgh Handedness Inventory (Oldfield, 1971). In no case was blindness associated with a central neural disorder. It is important to note that all of the participants had normal hand function, and in no case was blindness due to diabetic retinopathy (which may result in peripheral neuropathies in the hands; see Travieso \& Lederman, 2007).

\section{Haptic Line Bisection Task}

Stimuli consisted of wooden rods of four different lengths (300, 350,400 , and $450 \mathrm{~mm}$ ). The diameter of each rod was $14 \mathrm{~mm}$. Units of measurement $(\mathrm{mm})$ were marked with a pen on each rod to allow the experimenter to easily measure the distance between the participant's midpoint estimate and the actual midpoint after each trial. These marks could not be haptically perceived. The rods were positioned horizontally and centrally, with respect to the midline of the participants. Each rod was fixed with Velcro strips (attached on the bottom of each rod) horizontally onto a wooden panel. The rods could thus be explored without being moved, and a constant alignment between the participant's midsternum and the midpoint of the rod could be maintained. The distance between the subject's midsternum and the midpoint of the rod was maintained at about $380 \mathrm{~mm}$.

Sighted participants were blindfolded throughout the entire experiment. The participants were instructed to explore the length of the rod in their preferred direction (left to right or right to left), using the right index finger only. At the beginning of each trial, the experimenter placed the palm of the participant's right hand on the rod, so that it covered the midpoint of the rod, but the middle of the palm could be either slightly to the left or right of the rod's midpoint. This starting point for haptic exploration was used in order to avoid systematic biases in scanning direction, which are induced when exploration begins at either end of the rod. These systematic biases have been shown to influence bisection performance (cf. Baek et al., 2002). This palm-based starting position could not be used as an accurate estimate of the rod's midpoint, due to its approximate nature and because, at the start of each trial, the participants were asked to lift the palm off the rod and to begin to explore it with the right index finger. On each trial, the participants were given $10 \mathrm{sec}$ to scan the rod, and they could do so as many times as they wished. At the end 
of the trial, they were asked to indicate (with the right index finger) the midpoint of the rod. At the start of the experiment, a vertical line (approximately $1 \mathrm{~mm}$ wide) was drawn with a pen in the middle of the tip of the participants' right index fingers. After each trial, the experimenter used this line to measure the difference between the participants' perceived midpoint and the actual midpoint, within the nearest millimeter. For each of the four lengths of rod, there were 3 trials for each number condition (2, 8, and blah; see below), for a total of 36 trials. The number conditions, as well as different lengths of rods, were presented in random order. In each trial, the initial scanning direction (i.e., whether the participants started moving the finger toward the left or the right end of the rod) was recorded by the experimenter.

\section{Number Presentation}

On each trial, the participants were presented, in the auditory modality, with either a small (2) or a large (8) number, corresponding to the low and high ends of the mental number line, respectively. These numbers were chosen according to previous studies (e.g., Fischer, 2001; Fischer, Castel, Dodd, \& Pratt, 2003). This number was repeated 10 times, with a frequency of one per second (i.e., it lasted throughout the 10 -sec trial). On each trial, the start of the auditory stimulus was simultaneous with the start of the haptic exploration period. A control condition, in which participants were presented with the nonword "blah," was included in order to provide a baseline measure for any effects observed in the number conditions. Audio files were recorded using a specific sound program and were spoken by a female voice.

\section{Procedure}

The experiment started with a practice session (not included in the analyses), in which the participants were instructed to bisect each of the four lengths of rods within the 10 -sec time limit. The same auditory stimulus (blah) used in the control condition accompanied the exploration of the rod during this practice. The entire experiment lasted approximately $45 \mathrm{~min}$. No feedback was given to the participants during the testing.

\section{RESULTS}

As has been done in previous studies (e.g., Laeng, Buchtel, \& Butter, 1996), deviations from the veridical center were converted to signed percentage scores (positive if bisections were to the right, negative if to the left) by subtracting the true half-length of the rod from the measured distance of each setting from the left extremity of the rod, and then dividing this value by the true half-length and multiplying the quotient by 100 .

We used $t$ tests to compare averaged bias scores in the control (blah) condition for each group against zero (i.e.,

\section{A}

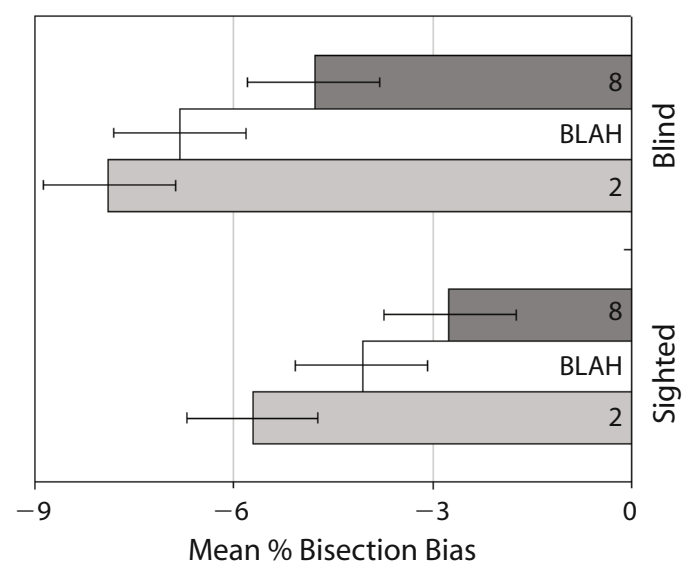

B

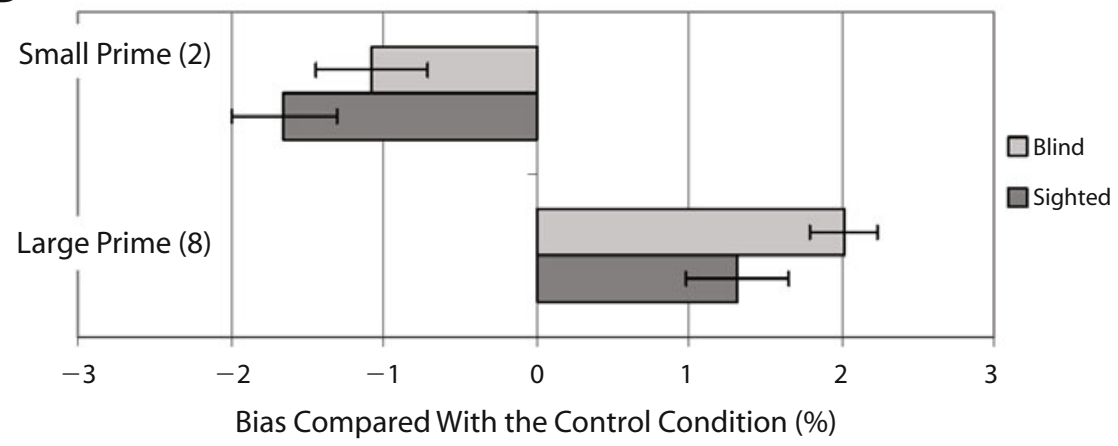

Figure 1. (A) Mean percentages of the biases of blind $(n=17)$ and sighted $(n=23)$ participants in bisecting rods in the control (blah), small-number (2), and large-number (8) conditions. The error bars indicate $\pm 1 S E M$. (B) Mean percentages of the biases of blind and sighted participants in bisecting rods in the small-number and large-number conditions after adjusting for biases in the control condition (i.e., the biases in the blah condition have been subtracted by each number condition, so that the resulting bias depends entirely on the number being presented and not on verbal stimulation itself). The error bars indicate $\pm 1 S E M$. 
the real midpoint value). Signed percentage deviations for the four different lengths were collapsed together in this analysis. Overall, both sighted and blind participants showed a tendency to bisect to the left of the veridical midpoint $[t(22)=3.39, p<.005$, and $t(16)=4.69, p<$ .001 , respectively] (see Figure 1A).

A mixed repeated measures ANOVA, with condition $(2,8$, or blah) and rod length as within-subjects variables and group (sighted vs. blind) as a between-subjects variable was performed on the signed percentage scores. The analysis revealed a significant main effect of condition $\left[F(2,76)=18.12, p<.001, \eta^{2}=.32\right]$ and a significant main effect of rod length $\left[F(3,114)=3.37, p<.05, \eta^{2}=\right.$ .08]. Pairwise comparisons revealed that the small number (2) shifted the perceived midline significantly to the left, as compared with the control (blah) condition $[t(39)=$ $2.81, p<.01]$ and that the large number (8) shifted the perceived midline significantly to the right, as compared with the control (blah) condition $[t(39)=3.72, p<.005]$ (see Figure 1B). The significant effect of rod length was due to the bisection bias being smaller overall for the $350-\mathrm{mm}$ rod than for the 400-mm rod $[t(39)=3.78, p<$ $.005]$. Neither group nor other interactions were significant. Notably, although the leftward bias in the large number condition was smaller than in the baseline condition, the estimated midline was still significantly shifted to the left of the rods' true midline for both sighted and blind participants $[t(22)=2.1, p<.05$, and $t(16)=3.17, p<$ .01 , respectively].

Possible differences in the initial scanning direction induced by the number's magnitude were also analyzed. Leftward scanning movements were assigned the code " -1 " and rightward scanning movements were assigned the code " +1 ." Hence, the sum of the starting scanning directions for each condition was computed. Overall, both sighted and blind participants showed a tendency to start exploring the rod to the left $[t(22)=3.32, p<.005$, and $t(16)=2.53, p<.05$, respectively]. A repeated measures ANOVA with condition as a within-subjects variable and group as a between-subjects variable was performed on the starting scanning directions scores. No significant main effect of condition ( $p=.37, \eta^{2}=.03$ ), of group $\left(p=.76, \eta^{2}=.002\right)$, or of the group $\times$ condition interaction $\left(p=.37, \eta^{2}=.03\right)$ was reported, indicating that 2 and 8 did not significantly affect the initial scanning direction, as compared with the blah condition.

\section{DISCUSSION}

Our findings demonstrate that number presentation can influence spatial judgments in a crossmodal auditoryto-haptic paradigm and that the interaction between the mental number line and peripersonal haptic space occurs, regardless of a lack of any previous visual experience. Specifically, in both the early-blind and blindfolded sighted participants, we found that listening to a small number (2) while haptically exploring a horizontal rod shifted its perceived midline significantly toward the left, as compared with a control condition, in which no numbers were presented. Conversely, a large number (8) shifted the per- ceived midpoint to the right, relative to the control condition, in all participants. This pattern of results is consistent with data obtained in the visual modality, showing that the low and high ends of the mental number line shift attention to the left and right side of space, respectively (e.g., Bonato et al., 2008; Dehaene et al., 1993; Fischer et al., 2003). Moreover, the present work critically extends previous findings on the effect of visually presented numbers on passive tactile stimulation (see Brozzoli et al., 2008) by showing the effect of auditorily presented numbers on active, haptic perception of space.

It is worth noting that numerical magnitude affected the participants' performance, even though number presentation was completely unrelated to the task. This is perhaps surprising, given evidence that suggests that the number needs to be task relevant in order to affect spatial attention (e.g., Galfano, Rusconi, \& Umiltà, 2006; Ristic, Wright, $\&$ Kingstone, 2006). However, others have found that simply perceiving a digit causes an automatic shift in spatial attention in a manner that is consistent with that digit's relative position on a mental number line (e.g., Fischer et al., 2003).

It is important to note that, in all three conditions (small number, large number, and control), the participants tended to bisect the rods to the left of the actual midpoint and started the scanning toward the left side of the rod. The tendency of bisecting to the left of the veridical center is usually referred to as pseudoneglect (cf. Bowers \& Heilman, 1980), and it has been reported both in the visual and the haptic modality (for a review, see Jewell \& McCourt, 2000). Pseudoneglect is believed to reflect an attentional asymmetry biased in favor of the left side of space, resulting from the dominant role of the right hemisphere in the allocation and control of spatial attention (McCourt \& Jewell, 1999; Mesulam, 2000). In fact, the direction of this bias is the opposite of that displayed in neglect patients, who typically suffer from a lesion to the right inferior parietal lobule or to the right temporo-parietal junction (cf. Vallar, 2001). The present study critically shows that the presence of pseudoneglect is not dependent on prior visual experience. Incidentally, it is worth noting that we found an effect of rod length due to an increase in the percentage bias between the 350- and 400-mm rods; however, it would not be entirely appropriate to conclude that the bias increased because of the increase of the rod length, since no difference was reported between the other rod lengths. Accordingly, previous findings are not consistent on this, since some studies have found an effect of rod length on the bisection bias (e.g., Laeng et al., 1996) and others have not (e.g., Baek et al., 2002).

Furthermore, our results confirm that the mental number line is oriented left to right in the blind, as it is in the sighted (Castronovo \& Seron, 2007). The left-to-right orientation of the mental number line has been associated in sighted individuals to reading habits (see Shaki, Fischer, \& Petrusic, 2009). Braille is also read from left to right (as are the Western languages), and this might have determined the left-to-right orientation of the mental number line in our blind participants (all proficient Braille readers). 
Since the rods were positioned centrally to the midline of the participants, it cannot be excluded that both blind and sighted individuals relied on a body-centered representation of space in performing the task, using their body midline as a reference for distance judgments. Previous studies suggest that blind individuals rely on egocentric space representation in perception of near space (see Gaunet \& Rossetti, 2006; Postma, Zuidhoek, Noordzij, \& Kappers, 2008), whereas sighted individuals tend to shift from an egocentric reference frame to an allocentric reference frame after a few seconds (see Rossetti, 1998; Rossetti \& Pisella, 2002; Rossetti \& Régnier, 1995; Zuidhoek, Kappers, van der Lubbe, \& Postma, 2003), and it has been demonstrated that the adoption of an egocentric versus an allocentric spatial reference frame may modulate the direction of numerical magnitude representation (e.g., Conson, Mazzarella, \& Trojano, 2009). On the basis of our findings, it is not possible to disentangle whether the rods' lengths were encoded into an egocentric or an allocentric reference frame; what is critical is that the mental number line was found to modulate the perception of haptic space in a crossmodal fashion. Future studies are needed to determine whether this effect is affected by reference frame manipulations-for instance, by presenting the target stimuli in the radial plane or in one hemifield in order to discourage the use of the body midline as a spatial reference.

Finally, our results with the early-blind participants suggest that spatial shifts of attention induced by a number's magnitude do not need the activation of a common, visually based representation to occur, but they are likely mediated by supramodal spatial features (see Struiksma, Noordzij, \& Postma, 2009) shared by both the mental number line and the representation of peripersonal haptic space.

To summarize, although previous evidence has shown that numbers are represented in a spatial format and that the mental number line can affect bimanual motor responses (i.e., the SNARC effect) regardless of the individual visual experience (Castronovo \& Seron, 2007; Szücs \& Csépe, 2005), our study provides the first evidence that the mental number line influences the allocation of spatial attention crossmodally in haptic peripersonal space and that it does so in a similar fashion in sighted and earlyblind individuals.

\section{AUTHOR NOTE}

We are grateful to Unione Italiana Ciechi e Ipovedenti, Susan Schmidt, and Antonella Di Stilo for their help in participants' recruitment. This work has been supported by Fondazione Banca del Monte di Pavia. Correspondence concerning this article should be addressed to Z . Cattaneo, Department of Psychology, University of Milano-Bicocca, Piazza dell'Ateneo Nuovo, Milano, Italy (e-mail: zaira.cattaneo@unimib.it).

\section{REFERENCES}

Baek, M. J., Lee, B. H., Kwon, J. C., Park, J. M., Kang, S. J., Chin, J., ET AL. (2002). Influence of final search direction on tactile line bisection in normal subjects. Neurology, 58, 1833-1838.

Bonato, M., Priftis, K., Marenzi, R., \& Zorzi, M. (2008). Modulation of hemispatial neglect by directional and numerical cues in the line bisection task. Neuropsychologia, 46, 426-433.

Bowers, D., \& Heilman, K. M. (1980). Pseudoneglect: Effects of hemispace on a tactile line bisection task. Neuropsychologia, 18, 491-498.
Brozzoli, C., Ishihara, M., Göbel, S. M., Salemme, R., Rossetti, Y., \& FARNÈ, A. (2008). Touch perception reveals the dominance of spatial over digital representation of numbers. Proceedings of the National Academy of Sciences, 105, 5644-5648.

Castronovo, J., \& Seron, X. (2007). Semantic numerical representation in blind subjects: The role of vision in the spatial format of the mental number line. Quarterly Journal of Experimental Psychology, 60, 101-119.

Cattaneo, Z., Silvanto, J., Pascual-Leone, A., \& Battelli, L. (2009). The middle range of the number line orients attention to the left side of visual space. Cognitive Neuropsychology, 26, 235-246.

Collignon, O., Voss, P., Lassonde, M., \& Lepore, F. (2009). Crossmodal plasticity for the spatial processing of sounds in visually deprived subjects. Experimental Brain Research, 192, 343-358.

Conson, M., Mazzarella, E., \& Trojano, L. (2009). Numbers are represented in egocentric space: Effects of numerical cues and spatial reference frames on hand laterality judgements. Neuroscience Letters, 452, 176-180.

Dehaene, S., Bossini, S., \& Giraux, P. (1993). The mental representation of parity and number magnitude. Journal of Experimental Psychology, 122, 371-396.

de Hevia, M. D., Girelli, L., \& Vallar, G. (2006). Numbers and space: A cognitive illusion? Experimental Brain Research, 168, 254-264.

FISCHER, M. H. (2001). Number processing induces spatial performance biases. Neurology, 57, 822-826.

Fischer, M. H., Castel, A. D., Dodd, M. D., \& Pratt, J. (2003). Perceiving numbers causes spatial shifts of attention. Nature Neuroscience, 6, 555-556.

Galfano, G., Rusconi, E., \& Umiltì, C. (2006). Number magnitude orients attention, but not against one's will. Psychonomic Bulletin \& Review, 13, 869-874.

Gaunet, F., \& Rossetti, Y. (2006). Effects of visual deprivation on space representation: Immediate and delayed pointing toward memorised proprioceptive targets. Perception, 35, 107-124.

Jewell, G., \& McCourt, M. E. (2000). Pseudoneglect: A review and meta-analysis of performance factors in line bisection tasks. Neuropsychologia, 38, 93-110.

LAeng, B., Buchtel, H. A., \& Butter, C. M. (1996). Tactile rod bisection: Hemispheric activation and sex differences. Neuropsychologia, 34, 1115-1121.

McCourt, M. E., \& Jewell, G. (1999). Visuospatial attention in line bisection: Stimulus modulation of pseudoneglect. Neuropsychologia, 37, 843-855.

Mesulam, M. M. (2000). Principles of behavioral and cognitive neurology (2nd ed.). Oxford: Oxford University Press.

Nicholls, M. E. R., Loftus, A. M., \& Gevers, W. (2008). Look, no hands: A perceptual task shows that number magnitude induces shifts of attention. Psychonomic Bulletin \& Review, 15, 413-418.

Nuerk, H.-C., WoOD, G., \& WiLlmes, K. (2005). The universal SNARC effect: The association between number magnitude and space is amodal. Experimental Psychology, 52, 187-194.

OlDFIELD, R. C. (1971). The assessment and analysis of handedness: The Edinburgh inventory. Neuropsychologia, 9, 97-113.

Postma, A., Zuidhoek, S., NoordziJ, M. L., \& Kappers, A. M. L. (2008). Haptic orientation perception benefits from visual experience: Evidence from early-blind, late-blind, and sighted people. Perception \& Psychophysics, 70, 1197-1206.

PutZar, L., Goerendt, I., Lange, K., Rösler, F., \& RöDer, B. (2007). Early visual deprivation impairs multisensory interactions in humans. Nature Neuroscience, 10, 1243-1245.

Ristic, J., Wright, A., \& Kingstone, A. (2006). The number line effect reflects top-down control. Psychonomic Bulletin \& Review, 13, $862-868$.

RöDER, B., FöcKer, J., Hötting, K., \& Spence, C. (2008). Spatial coordinate systems for tactile spatial attention depend on developmental vision: Evidence from event-related potentials in sighted and congenitally blind adult humans. European Journal of Neuroscience, 28, 475-483.

Röder, B., Kusmierek, A., Spence, C., \& Schicke, T. (2007). Developmental vision determines the reference frame for the multisensory control of action. Proceedings of the National Academy of Sciences, 104, 4753-4758.

RöDER, B., Rösler, F., \& Spence, C. (2004). Early vision impairs tactile perception in the blind. Current Biology, 14, 121-124. 
RosSeTtI, Y. (1998). Implicit short-lived motor representations of space in brain damaged and healthy subjects. Consciousness \& Cognition, 7, 520-558.

Rossetti, Y., \& Pisella, L. (2002). Several "vision for action" systems: A guide to dissociating and integrating dorsal and ventral functions. In W. Prinz \& B. Hommel (Eds.), Attention and performance XIX: Common mechanisms in perception and action (pp. 62-119). New York: Oxford University Press.

Rossetti, Y., \& RÉGnIER, C. (1995). Representations in action: Pointing to a target with various representations. In B. G. Bardy, R. J. Bootsma, \& Y. Guiard (Eds.), Studies in perception and action III (pp. 233-236). Mahwah, NJ: Erlbaum.

Shaki, S., Fischer, M. H., \& Petrusic, W. M. (2009). Reading habits for both words and numbers contribute to the SNARC effect. Psychonomic Bulletin \& Review, 16, 328-331.

Struiksma, M. E., NoordziJ, M. L., \& Postma, A. (2009). What is the link between language and spatial images? Behavioral and neural findings in blind and sighted individuals. Acta Psychologica, 132, 145-156.

SzÜCS, D., \& CsÉPE, V. (2005). The parietal distance effect appears in both the congenitally blind and matched sighted controls in an acoustic number comparison task. Neuroscience Letters, 384, 11-16.
Travieso, D., \& Lederman, S. J. (2007). Assessing subclinical tactual deficits in the hand function of diabetic blind persons at risk for peripheral neuropathy. Archives of Physical Medicine \& Rehabilitation, 88, 1662-1672.

Umiltà, C., Priftis, K., \& ZorZi, M. (2009). The spatial representation of numbers: Evidence from neglect and pseudoneglect. Experimental Brain Research, 192, 561-569.

VALLAR, G. (2001). Extrapersonal visual unilateral spatial neglect and its neuroanatomy. NeuroImage, 14, S52-S58.

Wood, G., Nuerk, H. C., \& Willmes, K. (2006). Crossed hands and the SNARC effect: A failure to replicate Dehaene, Bossini and Giraux (1993). Cortex, 42, 1069-1079.

Wood, G., Willmes, K., Nuerk, H.-C., \& Fischer, M. H. (2008). On the cognitive link between space and number: A meta-analysis of the SNARC effect. Psychology Science Quarterly, 50, 489-525.

Zuidhoek, S., Kappers, A. M. L., van der Lubbe, R. H. A., \& Postma, A. (2003). Delay improves performance on a haptic spatial matching task. Experimental Brain Research, 149, 320-330.

(Manuscript received November 11, 2009; revision accepted for publication January 19, 2010.) 